\title{
Intercalation and Stitching of Graphite Oxide with Diaminoalkanes
}

\author{
Margarita Herrera-Alonso, Ahmed A. Abdala, ${ }^{\dagger}$ Michael J. McAllister, Ilhan A. Aksay, and \\ Robert K. Prud'homme*
}

Department of Chemical Engineering, Princeton University, Princeton, New Jersey 08544

Received June 7, 2007

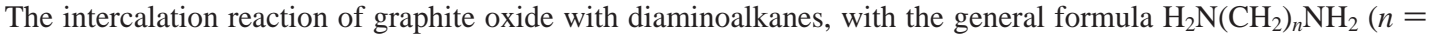
4-10), was studied as a method for synthesizing pillared graphite with tailored interlayer spacing. Interlayer spacings from 0.8 to $1.0 \mathrm{~nm}$ were tailored by varying the size of the intercalant from $\left(\mathrm{CH}_{2}\right)_{4}$ to $\left(\mathrm{CH}_{2}\right)_{10}$. X-ray diffraction and infrared spectroscopy were used to confirm intercalation, and the frequency of the $\mathrm{CH}_{2}$ stretch confirmed that the intercalants are in a disordered state, with an important contribution from the gauche conformer. Sequential intercalation of diaminoalkanes followed by dodecylamine demonstrated the inability of these "stitched" systems to undergo expansion along the $c$-direction, indicative of cross-linking. Finally, the reaction of graphite oxide with diaminoalkanes under reflux and for extended periods $(>72 \mathrm{~h}$ ) resulted in the chemical reduction of the graphite oxide to a disordered graphitic structure.
\end{abstract}

\section{Introduction}

Intercalation of organic molecules in the gallery spacing of layered materials is a common modification technique since the simplicity of these reactions allows facile control over the chemical and physicochemical characteristics of the derivatives. ${ }^{1}$ An example of the applicability of this method is in the fabrication of polymer/clay nanocomposites, where dispersability of the inorganic platelets within the polymer matrix has a profound effect on the mechanical, thermal, and barrier properties of the resulting composite. ${ }^{2}$ Organophilization of inherently hydrophilic aluminosilicates through ion-exchange reactions with alkylammonium quaternary salts results in surfaces that exhibit enhanced compatibility with hydrophobic polymers, therefore improving their dispersability. ${ }^{3}$ Recently much attention has been focused on intercalation of difunctional reagents since they can lead to bridged or pillared nanostructures with potential applications as catalytic supports, selective adsorbents, and molecular vessels. ${ }^{4-6}$

Depending on the nature of the host and the intercalant, pillaring and stitching can be accomplished by three methods: (i) ion exchange between a difunctional ionic intercalant and the native ions of the host; (ii) exfoliation of the host followed by reaction with the difunctional reagent and reassembly; and (iii) intercalation of a difunctional molecule chemically anchored at one end and capable of undergoing a different reaction on the other end. Noteworthy examples of bridged systems include $\gamma$-zirconium phosphate bridged with $n$-alkanediphosphonic acids or polyethylene oxide, ${ }^{4,5}$ montmorillonite (MMT) clay bridged by diaminoalkanes, ${ }^{6}$ and graphite oxide $(\mathrm{GO})$ pillared with (3aminopropyl)trimethoxysilane or iron oxide. ${ }^{7,8}$ Intercalation of a difunctional reagent, however, does not ensure bridging, since

\footnotetext{
* Corresponding author. E-mail: prudhomm@princeton.edu.

$\dagger$ Current address: Department of Chemical Engineering, The Petroleum Institute, POB 2533, Abu Dhabi, United Arab Emirates.

(1) Lagaly, G. Appl. Clay Sci. 1999, 15, 1-9.

(2) Gleiter, H. Adv. Mater. 1992, 4, 474-481. Novak, B. M. Adv. Mater. 1993, 5, 422-433. Pinnavaia, T. J.; Lan, T. Chem. Mat. 1994, 6, 2216-2219. Yano, K.; Usuki, A.; Okada, A.; Kurauchi, T.; Kamigaito, O. J. Polym. Sci., Part A: Polym. Chem. 1993, 31, 2493-2498.

(3) Messersmith, P. B.; Giannelis, E. P. Chem. Mat. 1994, 6, 1719-1725.

(4) Alberti, G.; Mascarós-Murcia, S.; Viviani, R. J. Am. Chem. Soc. 1998, 120 , 9291-9295.

(5) Alberti, G.; Brunet, E.; Dionigi, C.; Juanes, O.; de la Mata, M. J.; RodríguezUbis, J. C.; Viviani, R. Angew. Chem., Int. Ed. 1999, 38, 3351-3353.

(6) Ha, B.; Char, K. Langmuir 2005, 21, 8471-8477.
}

the intercalant can also adopt a single-tail tethered conformation, where only one end of the difunctional molecule reacts, or a loop conformation, where each end interacts with the same layer. ${ }^{6}$

$\mathrm{GO}$, an oxygen-rich derivative of graphite, is a layered material capable of undergoing intercalation by one-dimensional expansion along its $c$-axis. Since its first synthesis by Brodie in the $1850 \mathrm{~s},{ }^{9}$ GO has been characterized by a combination of spectroscopic techniques in order to establish a structural model that explains its chemical composition and reactivity. Although its stoichiometry depends on the method used for its preparation, it is commonly accepted that it consists of randomly distributed regions of unoxidized (aromatic) graphite and regions of aliphatic sixmembered rings, rich in oxygen-containing functional groups, including epoxys and hydroxyls. ${ }^{10-12}$ It is believed that the epoxy and hydroxyl functionalities lie above and below each layer, while carboxylic acid groups decorate the edges. ${ }^{10}$ Oxidation, therefore, provides the otherwise unreactive graphite with functional groups that can be used as reactive handles to tailor its properties. The hydroxyl groups possess acidic protons, allowing $\mathrm{GO}$ to undergo intercalation through ion exchange, analogous to MMT clays. ${ }^{13-16}$ However, unlike MMT, its rich surface chemistry also allows covalent bonding of the intercalant directly to the layers. Chemistries targeted at the hydroxy and epoxy functionalites have been studied previously, with the objective of assessing the reactivity of these functional groups in the intercalated structure, as well as controlling the interlayer spacing and hydrophobicity by careful consideration of the size and molecular structure of the intercalants. $7,10,17-19$

(7) Bourlinos, A. B.; Gournis, D.; Petridis, D.; Szabó, T.; Szeri, A.; Dékány, I. Langmuir 2003, 19, 6050-6055

(8) Morishige, K.; Hamada, T. Langmuir 2005, 21, 6277-6281.

(9) Brodie, B. C. Philos. Trans. R. Soc. London 1859, 149, 249

(10) Lerf, A.; He, H.; Forster, M.; Klinowski, J. J. Phys. Chem. B 1998, 102, 4477-4482

(11) Mermoux, M.; Chabre, Y.; Rousseau, A. Carbon 1991, 29, 469-474.

(12) Hontoria-Lucas, C.; López-Peinado, A. J.; López-González, J. de D. Rojas-Cervantes, M. L.; Martín-Aranda, R. M. Carbon 1995, 33, 1585-1592. (13) Liu, Z.-H.; Wang, Z.-M.; Yang, X.; Ooi, K. Langmuir 2005, 18, 49264932.

(14) Matsuo, Y.; Niwa, T.; Sugie, Y. Carbon 1999, 37, 897-901.

(15) Matsuo, Y.; Watanabe, K.; Fukutsuka, T.; Sugie, Y. Carbon 2003, 41 $1545-1550$.

(16) Dékány, I.; Krüger-Grasser, R.; Weiss, A. Colloid Polym. Sci. 1998, 276, $570-576$.

(17) Matsuo, Y.; Fukunaga, T.; Fukutsuka, T.; Sugie, Y. Carbon 2004, 42, $2113-2130$ 
An interesting and potentially important application of pillared graphitic materials is in the area of hydrogen storage. Recent theoretical calculations indicate that confinement effects should have a major impact on hydrogen storage between graphite sheets. ${ }^{20}$ Simulations show that there exists an optimal combination of pressure and pore geometry (interlayer distance) that maximizes the storage capacity. ${ }^{20}$ Furthermore, chemical substitution disrupts the planarity of the graphene sheets and creates a distorted surface, which has been referred to as "puckered" 21 or "roughened". 22 The combination of altered aromaticity, bond distortion, and local coordination changes arising from the distorted surface, cause an increase in the polarizability of graphene. Binding energies for hydrogen range from $52 \mathrm{meV}$ in a honeycomb graphene structure to $75 \mathrm{meV}$ in a model system containing Stone-Wales defects. ${ }^{22}$ Pillaring of the graphitic sheets would be necessary to maintain the optimal separation between graphite layers and to resist delamination of the layered structure during pressure cycling of a hydrogen storage medium.

The present work describes a detailed study of the intercalation reaction of $\alpha, \omega$-diaminoalkanes with GO to produce chemically bridged derivatives. The size of the intercalant was systematically varied to determine its effect on the interlayer spacing, and the conformation of the intercalant in the layered structure was assessed by swelling experiments.

\section{Experimental Section}

Materials. All materials were used as received. Graphite particles ( $40 \mu \mathrm{m}$ particle size) were provided by Asbury Carbon, New Jersey. Sulfuric acid (98\%), hydrochloric acid (37\%), and nitric acid (fuming, $90 \%$ ) were purchased from Fisher. Potassium chlorate, ethanol (reagent grade), diaminoalkanes, and alkylamines were obtained from Aldrich.

Graphite Oxide. GO was synthesized from natural flake graphite (40 $\mu \mathrm{m}$ particle size) by the Staudenmaier method. ${ }^{23}$ Sulfuric acid $(160 \mathrm{~mL})$ and nitric acid $(90 \mathrm{~mL})$ were added to a round-bottom flask, containing a stir bar, and cooled with an ice bath for $1 \mathrm{~h}$. Graphite particles (10 g) were added to the acids mixture under vigorous stirring, and the suspension was cooled for $20 \mathrm{~min}$. Potassium chlorate (110 g) was slowly added over $15 \mathrm{~min}$, while keeping the reaction vessel inside an ice bath, making sure that the temperature did not exceed $35^{\circ} \mathrm{C}$. Caution! This reaction results in the formation of chlorine dioxide gas, which is explosive at high concentrations. ${ }^{24}$ To minimize the risk of explosion it is recommended that the addition of potassium chlorate be done slowly while monitoring the temperature. Oxidation was allowed to proceed for $96 \mathrm{~h}$. The suspension was washed with an aqueous hydrochloric acid solution (10 vol \%) to remove the sulfate ions and then washed repeatedly with deionized (DI) water until neutral $\mathrm{pH}$. The final concentration of GO in the suspension was approximately $6 \mathrm{mg}$ $\mathrm{GO} / \mathrm{mL}$.

Intercalation of Graphite Oxide with Mono- and Difunctional Amines. In a typical experiment, 1,8-diaminooctane (600 mg) was dissolved in ethanol $(35 \mathrm{~mL})$ and added dropwise to a suspension of $\mathrm{GO}$ in water (200 mg GO, $33 \mathrm{~mL}$ ) under vigorous stirring. The

(18) Valerga Jiménez, P.; Arufe Martínez, M. I.; Martín Rodríguez, A. Carbon 1985, 23, 473-479.

(19) Aragón, F.; Ruíz, J. C.; MacEwan, D. M. C. Nature 1959, 183, 740-741. (20) Wang, Q.; Johnson, J. K. J. Chem. Phys. 1999, 110, 577-586. Wang, Q.; Johnson, J. K. J. Phys. Chem. B 1999, 103, 277-281. Kowalczyk, P.; Tanaka, H.; Holyst, R.; Kaneko, K.; Ohmori, T.; Miyamoto J. J. Phys. Chem. B 2005, 109, 17174-17183. Rzepka, M.; Lamp, P.; de la Casa-Lillo, M. A. J. Phys. Chem. B 1998, 102, 10894-10898.

(21) Boehm, H. P.; Setton, R.; Stumpp, E. Pure Appl. Chem. 1994, 66, $1893-$ 1901

(22) Pradhan, B. K.; Harutyunyan, A. R.; Stojkovic, D.; Grossman, J. C.; Zhang, P.; Cole, M. W.; Crespi, V.; Goto, H.; Fujiwara, J.; Eklund, P. C. J. Mater. Res. 2002, 17, 2209-2216.

(23) Staudenmaier, L. Ber. Dtsch. Chem. Ges. 1898, 31, 1481-1487.

(24) López, M. I.; Croce, A. E.; Sicre, J. E. J. Chem. Soc., Faraday Trans.

1994, 90, 3391-3396. reaction continued for $24 \mathrm{~h}$ at room temperature or under reflux $\left(78{ }^{\circ} \mathrm{C}\right)$. Intercalated $\mathrm{GO}$ was isolated by centrifugation and thoroughly washed with $1: 1$ ethanol/water $(45 \mathrm{~mL}, 4 \times)$, filtered, and dried at $80{ }^{\circ} \mathrm{C}$ under vacuum for a minimum of $12 \mathrm{~h}$ prior to characterization. Reactions with alkylamines $\mathrm{CH}_{3}\left(\mathrm{CH}_{2}\right)_{n} \mathrm{NH}_{2}(n=$ 4-12) or diaminoalkanes $\mathrm{H}_{2} \mathrm{~N}\left(\mathrm{CH}_{2}\right)_{n} \mathrm{NH}_{2}(n=4-10)$ were conducted following the same procedure.

Characterization. X-ray diffraction (XRD) patterns were acquired on a Rigaku MiniFlex diffractometer with $\mathrm{Cu} \mathrm{K}_{\alpha}$ radiation. Simultaneous thermogravimetric analysis (TGA) and differential scanning calorimetry (DSC) experiments were conducted with a Nezstch STA 449C thermal analyzer. Analyses were done under a nitrogen atmosphere $(50 \mathrm{~mL} / \mathrm{min}$ flow rate) at a heating rate of $1{ }^{\circ} \mathrm{C} / \mathrm{min}$. Fourier transform infrared spectroscopy (FTIR) was performed on a ThermoNicolet 6700 FT-IR spectrometer. GO and its derivatives were thoroughly ground, mixed with potassium bromide to a final concentration of approximately $0.15 \mathrm{wt} \%$, and pressed into pellets. Elemental analysis was performed by Atlantic Microlab Inc., Norcross GA.

\section{Results and Discussion}

Graphite Oxide Characterization. GO was characterized by elemental analysis, ${ }^{13} \mathrm{C}$ NMR, XPS, and FTIR. Results from XPS and elemental analysis were discussed in a previous publication. ${ }^{25}$ Briefly, the chemical composition of GO determined by elemental analysis was $\mathrm{C}_{8} \mathrm{O}_{4.3} \mathrm{H}_{3.6}$, while the $\mathrm{C} / \mathrm{O}$ ratio calculated from XPS was 2.6. High-resolution $\mathrm{C}_{1 \mathrm{~s}}$ XPS (Figure 1, Supporting Information) showed signals characteristic of graphitic carbon $(\mathrm{C}-\mathrm{C}, 284.6 \mathrm{eV})$ and carbon singly bound to oxygen $(\mathrm{C}-\mathrm{O}, 286.6 \mathrm{eV})$, either as epoxy or hydroxyl. ${ }^{12,26}$ The broad $\mathrm{O}_{1 \mathrm{~s}}$ spectrum is also indicative of the presence of more than one oxygen-containing species: oxygen in carboxyls $(531.3 \mathrm{eV}), \mathrm{C}_{\text {aliphatic }}-\mathrm{O}(532.6 \mathrm{eV})$, and $\mathrm{C}_{\text {aromatic }}-\mathrm{O}(531.1 \mathrm{eV}){ }^{12}$ Solid-state ${ }^{13} \mathrm{C}$ NMR results (Figure 2, Supporting Information) are in agreement with the XPS data. The spectrum contains two distinguishable peaks at chemical shifts $(\delta)$ of 60-70 and 133 ppm. The signal between 60 and $70 \mathrm{ppm}$ is composed of two peaks, which have been assigned to hydroxyl (70 ppm) and 1,2epoxy groups $(60 \mathrm{ppm})$. The peak at $133 \mathrm{ppm}$ corresponds to $\mathrm{sp}^{2}$-hybridized carbon. The third signal, observed at lower fields (210-220 ppm), has been assigned to spinning side bands of the unsaturated carbons. ${ }^{11}$ The NMR spectrum is in qualitative agreement with the model proposed by Lerf et al. ${ }^{10}$ FTIR analysis of GO is discussed in more detail further in the text.

Intercalation of Graphite Oxide with $\alpha, \omega$-Diaminoalkanes. The rich surface chemistry of GO allows for covalent modification by reactions at hydroxy and epoxy groups. An example is the intercalation reaction of $\mathrm{GO}$ with monofunctional amines, which proceeds by nucleophilic substitution on the epoxy groups, provided that the amines are in neutral form. ${ }^{7,10}$ It is possible then, that intercalation of a difunctional amine could produce a pillared structure, as shown in Figure 1.

For this, GO was reacted with a series of $\alpha, \omega$-diaminoalkanes that differ in the number of methylene units $(n)$ separating the terminal amine groups. XRD patterns of GO and the intercalated derivatives are shown in Figure 2. A gradual increase in the basal spacing of GO is observed as the hydrocarbon chain length of the diamine increases from $n=4$ to $n=10$, suggesting that intercalation takes place and that the interlayer spacing is sensitive to the size of the intercalant.

(25) Schniepp, H. C.; Li, J.-L.; McAllister, M. J.; Sai, H.; Herrera-Alonso, M.; Adamson, D. H.; Prud'homme, R. K.; Car, R.; Saville, D. A.; Aksay, I. A. J. Phys. Chem. B 2006, 110, 8535-8539.

(26) Xing, Y. C.; Li, L.; Chusuei, C. C.; Hull, R. V. Langmuir. 2005, 21, 4185-4190. de la Puente, G.; Pis, J. J.; Menendez, J. A.; Grange, P. J. Anal. Appl. Pyrolysis 1997, 43, 125-138. 


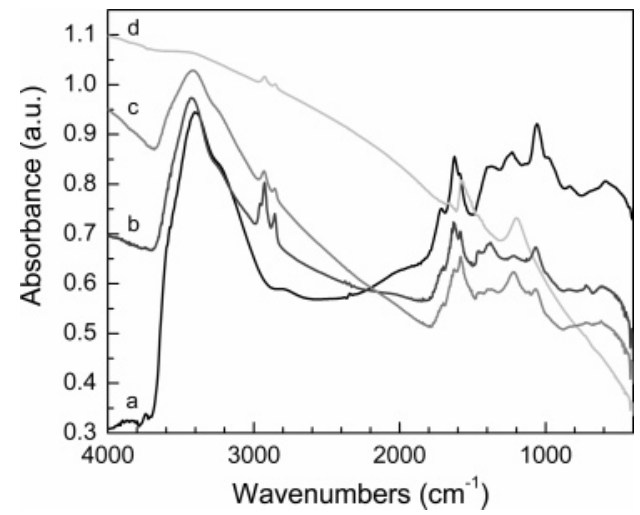

Figure 5. FTIR spectra of GO (a), GO/octylamine (b), GO/ diaminooctane at room temperature (c), GO/diaminooctane under reflux (d). The intercalation of mono- and difunctional amines is evidenced by the appearance of methylene bands $\left(2800-3000 \mathrm{~cm}^{-1}\right)$.

conditions. It is important to mention that this reaction was observed only in the presence of the diamine. While the mechanism for this unexpected reaction is unknown, we suspect that it could be attributed to the presence of trace amounts of metals in the diamine (purity $\sim 95 \%$ ), consistent with the deoxygenation of aromatic alcohols in refluxing 2-propanol and Raney nickel catalyst. ${ }^{29}$

Infrared spectroscopy was also used to confirm intercalation; FTIR spectra of GO and its derivatives are presented in Figure 5. The spectrum of GO is in good agreement with previous work. ${ }^{11,12,30}$ The intense band at $3408 \mathrm{~cm}^{-1}$ and the broad band at $3200 \mathrm{~cm}^{-1}$ are attributed to stretching of the $\mathrm{O}-\mathrm{H}$ bond of $\mathrm{CO}-\mathrm{H}$ and water, respectively. The band at $1700 \mathrm{~cm}^{-1}$ is associated with stretching of the $\mathrm{C}=\mathrm{O}$ bond of carbonyl or carboxyl groups. The bands present at 1630 and $1383 \mathrm{~cm}^{-1}$ are attributed to deformations of the $\mathrm{O}-\mathrm{H}$ bond in water and $\mathrm{CO}-\mathrm{H}$ groups, respectively. Deformation of the $\mathrm{C}-\mathrm{O}$ bond is observed as the intense band present at $1057 \mathrm{~cm}^{-1}$. The FTIR spectra of GO intercalated with octylamine, as reference, and diaminooctane (at room temperature) differ from that of GO in the signals observed at $1470,1570,2924$, and $2852 \mathrm{~cm}^{-1}$. The first two peaks are assigned to the bending vibration (scissoring) of $-\mathrm{CH}_{2}-$, and vibration of the $\mathrm{N}-\mathrm{H}$ groups of the intercalants, respectively. The strongest signals, observed at higher frequencies, are attributed to asymmetric $\left(2924 \mathrm{~cm}^{-1}\right)$ and symmetric $\left(2852 \mathrm{~cm}^{-1}\right)$ stretching of the methylene group of the alkylamines (spectra $\mathrm{b}$ and $\mathrm{c}$ of Figure 5). Similar results were observed for intercalants with $4<n<10$. The higher intensity of these bands for the monofunctional vs the difunctional amine is indicative of a larger amount of intercalated material for the former case.

Detailed studies on the intercalation of alkylamines in the interlayer spacing of MMT clays revealed that the frequency and intensity of the bending mode $(\delta)$, and asymmetric $\left(v_{\mathrm{as}}\right)$ and symmetric $\left(v_{\mathrm{s}}\right)$ stretching modes of the $\mathrm{CH}_{2}$ group are sensitive to the conformation and packing arrangement of the alkyl chains in confined environments. ${ }^{6,31-33}$ Interchain interactions and packing arrangements can be inferred from the intensity and shape of the bending vibration, observed between 1466 and $1472 \mathrm{~cm}^{-1}$. Higher frequencies are indicative of all-trans chains

(29) Gross, B. H.; Mebane, R. C.; Armstrong, D. L. Appl. Catal., A 2001, 219 , $281-289$.

(30) Kovtyukhova, N. I.; Olliver, P. J.; Martin, B. R.; Mallouk, T. E.; Chizhik, S. A.; Buzaneva, E. V.; Gorchinskiy, A. D. Chem. Mater. 1998, 11, 771-778. (31) Vaia, R. A.; Teukolsky, R. K.; Giannelis, E. P. Chem. Mater. 1994, 6 , 1017-1022.

(32) Li, Y.; Ishida, H. Langmuir 2003, 19, 2479-2484.

(33) Xie, W.; Gao, Z.; Pan, W.-P.; Hunter, D.; Singh, A.; Vaia, R. Chem. Mater. 2001, 13, 2979-2990. in a crystalline state. Increased chain motion, associated with a less-ordered liquid-like state, leads to peak broadening and decreased intensities. On the other hand, chain conformation (gauche/trans ratio) can be estimated from the asymmetric stretching bands with frequencies ranging from 2937 to $2919 \mathrm{~cm}^{-1}$. In general, a shift in $v_{\text {as }}\left(\mathrm{CH}_{2}\right)$ from a lower frequency, indicative of highly ordered all-trans systems, to a higher value, is observed as the number of gauche conformers, and thus the disorder, increases. The reported value of $v_{\text {as }}$ for fully stretched chains was $2919 \mathrm{~cm}^{-1},{ }^{31}$ while the most disordered system with the highest gauche/trans conformer ratio was observed at $2937 \mathrm{~cm}^{-1}$.6,32 Bending and asymmetric stretching of the methylene group of GO intercalated with diaminooctane and octylamine occurs at $\delta=1467 \mathrm{~cm}^{-1}$ and $\nu_{\mathrm{as}}=2929 \mathrm{~cm}^{-1}$, and $\delta=1466 \mathrm{~cm}^{-1}$ and $\nu_{\text {as }}=2926 \mathrm{~cm}^{-1}$, respectively. Compared to reference values, the broadness of the bending signal, in combination with the peak position of the asymmetric stretching suggests that the intercalants are in a disordered state, with an important contribution from the gauche conformer. This observation also explains the difference between the experimental and theoretical interlayer spacing (Figure 3), as the premise of a chain-extended conformation is not applicable. Furthermore, the difference in $v_{\text {as }}$ peak positions between the difunctional and monofunctional intercalants suggests a higher degree of conformational order for the monofunctional case.

The spectrum of GO intercalated with diaminoalkanes under reflux for an extended period $(72 \mathrm{~h}$ ) is essentially featureless and markedly different from that of its precursor. The signals characteristic of oxygen-containing functionalities are lost, and two new signals, at 1572 and $1199 \mathrm{~cm}^{-1}$ appear (Figure 5d). This spectrum is reminiscent of pristine graphite, which has two IRactive modes: a signal at $1571 \mathrm{~cm}^{-1}$, attributed to the in-plane $\mathrm{E}_{1 \mathrm{u}}$ mode, and a signal at $868 \mathrm{~cm}^{-1}$, assigned to the out-of-plane $\mathrm{A}_{2 \mathrm{u}}$ mode. ${ }^{34-37}$ The latter signal is not observed in the spectra presented in Figure 5 since it is generally of lower intensity and difficult to observe. The signal at $1571 \mathrm{~cm}^{-1}$ shows broadening toward lower energy, which has been attributed to increased disorder, bending of the graphite sheets, and a change in the interplanar bonding. ${ }^{36}$ The peak at $1194 \mathrm{~cm}^{-1}$ was previously observed for chemical vapor-deposited single-wall carbon nanotubes; however, it has not yet been identified. ${ }^{37}$ These results support those from XRD in that, while intercalation of the difunctional amines occurs, more vigorous reaction conditions result in the chemical reduction of GO. This suggests that reduction produces a collapse from the original $0.68 \mathrm{~nm} \mathrm{GO}$ spacing to turbostatic graphite with $\sim 0.4 \mathrm{~nm}$ spacing.

The thermal behavior of GO and its derivatives was studied by TGA/DSC (Figure 6). GO, hydrophilic because of its high concentration of polar functional groups, exhibits two regions of mass loss. The first occurs at temperatures below $125^{\circ} \mathrm{C}$ and is attributed to loss of adsorbed water $(\sim 4 \%)$. The second occurs at an onset temperature of $150{ }^{\circ} \mathrm{C}$ and is associated with the thermal decomposition of oxygen-containing functionalities to generate carbon dioxide and water.7,13,30,38 The corresponding DSC shows a large exothermic signal with a peak at $210^{\circ} \mathrm{C}$. The curve slowly plateaus around $300{ }^{\circ} \mathrm{C}$, with a total mass loss of

(34) Friedel, R. A.; Carlson, G. L. J. Phys. Chem. 1971, 75, 1149-1151.

(35) Conrad, M. P.; Strauss, H. L. Phys. Rev. B 1985, 31, 6669-6675.

(36) Kastner, J.; Pichler, T.; Kuzmany, H.; Curran, S.; Blau, W.; Weldon, D. N.; Delamesiere, M.; Draper, S.; Zandbergen, H. Chem. Phys. Lett. 1994, 221 $53-58$.

(37) Zhang, J.; Zou, H.; Qing, Q.; Yang, Y.; Li, Q.; Liu, Z.; Guo, X.; Du, Z. J. Phys. Chem. B 2003, 107, 3712-3718.

(38) McAllister, M. J.; Li, J.-L.; Adamson, D. H.; Schniepp, H. C.; Abdala, A. A.; Liu, J.; Herrera-Alonso, M.; Milius, D. L.; Car, R.; Prud'homme, R. K.; Aksay, I. A. Chem. Mater. Published online May 25, 2007, http://dx.doi.org/ $1021 / \mathrm{cm} 0630800$ 


\title{
Raman Spectra of Graphite Oxide and Functionalized Graphene Sheets
}

\author{
Konstantin N. Kudin, ${ }^{\dagger}$ Bulent Ozbas, ${ }^{\ddagger}$ Hannes C. Schniepp,, \\ Robert K. Prud'homme, ${ }^{\ddagger}$ Ilhan A. Aksay, ${ }^{\ddagger}$ and Roberto Car ${ }^{\star}, \dagger$ \\ Department of Chemistry, and Department of Chemical Engineering, \\ Princeton University, Princeton, New Jersey 08544
}

Received July 26, 2007; Revised Manuscript Received October 25, 2007

\begin{abstract}
We investigate Raman spectra of graphite oxide and functionalized graphene sheets with epoxy and hydroxyl groups and Stone-Wales and 5-8-5 defects by first-principles calculations to interpret our experimental results. Only the alternating pattern of single-double carbon bonds within the $\mathrm{sp}^{2}$ carbon ribbons provides a satisfactory explanation for the experimentally observed blue shift of the $\mathrm{G}$ band of the Raman spectra relative to graphite. To obtain these single-double bonds, it is necessary to have $\mathrm{sp}^{3}$ carbons on the edges of a zigzag carbon ribbon.
\end{abstract}

Motivated by the promise of graphene as an alternative to single-wall carbon nanotubes, in recent studies, we reported ${ }^{1,2}$ a method to produce functionalized single graphene sheets (FGSs) in bulk quantities through thermal expansion of graphite oxide (GO). The aromaticity of the graphene sheets is lost as epoxide and hydroxyl groups are also formed during the oxidation process, resulting in an interlayer spacing ( $d$ spacing) increase from 0.34 to $\sim 0.7 \mathrm{~nm} .{ }^{1}$ Pressure buildup due to $\mathrm{CO}_{2}$ release during rapid heating separates stacked GO layers to produce functionalized single graphene sheets. While the $\mathrm{C} / \mathrm{O}$ ratio of GO is $\sim 2$, the FGS produced by this approach has a $\mathrm{C} / \mathrm{O}$ ratio of $10-20$ as some of the epoxide and hydroxyl sites are eliminated. ${ }^{2}$

Both from a practical and a fundamental perspective, it is important to understand the nature of disorder in GO and FGSs. Despite the fact that GO has been known since 1859 and several GO models ${ }^{3-7}$ have been suggested, its detailed structure is still under debate, and a definitive atomistic model is not yet available. For a detailed understanding of mechanical and electronic properties of the FGS, we would like to know (i) if the honeycomb carbon network of graphene remains essentially intact or if rings other than sixmembered rings and other topological defects appear in the oxidation process; (ii) if the epoxy and hydroxyl groups are distributed randomly, if they retain some amount of short/ intermediate range order, or if they tend to cluster, creating patches of more strongly oxidized graphene intermixed with patches of near-perfect graphene; and (iii) if a portion of the defect anneals during the thermal treatment process.

* To whom correspondence should be addressed. E-mail: rcar@ princeton.edu.

Department of Chemistry, Princeton University.

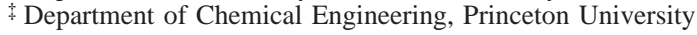

To provide answers to some of these questions, here, we report our work on the characterization of GO and FGSs by Raman spectroscopy and scanning tunneling microscopy (STM). We also report calculated vibrational spectra of various functional fragments that might appear during the chemical transformation of graphite to determine which spectroscopic features appear for a particular atomic arrangement. Specifically, using plane wave density functional theory $(\mathrm{DFT})^{8}$ calculations, we have computed the Raman spectra of graphene sheets with defects, as well as epoxy and hydroxyl groups attached in various configurations. The presence of such groups in GO was deduced from NMR spectroscopy and group-specific reaction chemistry by Lerf et al. ${ }^{7}$ In the processing of similar materials — carbon nanotubesoxidation also plays an important role when purifying nanotubes in order to remove the amorphous carbon. ${ }^{9}$

Raman spectroscopy is a widely used tool for the characterization of carbon products, especially considering the fact that conjugated and double carbon-carbon bonds lead to high Raman intensities. However, we are aware of only one previous computational study that investigated the Raman spectra of oxidized nanotubes modeled by relatively short nanotube segments terminated with hydrogen. ${ }^{10}$ This geometry introduced spurious features into the vibrational modes, making the interpretation of the experimental spectroscopic features somewhat challenging. Another work ${ }^{11}$ calculated the infrared spectra of various finite graphitic structures with edge groups. Since most GO models involve a mostly intact hexagonal carbon lattice, placing chemical groups randomly throughout the $2 \mathrm{D}$ sheet and not on the edges should model the GO structure more accurately.

Highly ordered graphite has only a couple of Raman-active bands visible in the spectra (Figure 1), the in-phase vibration 


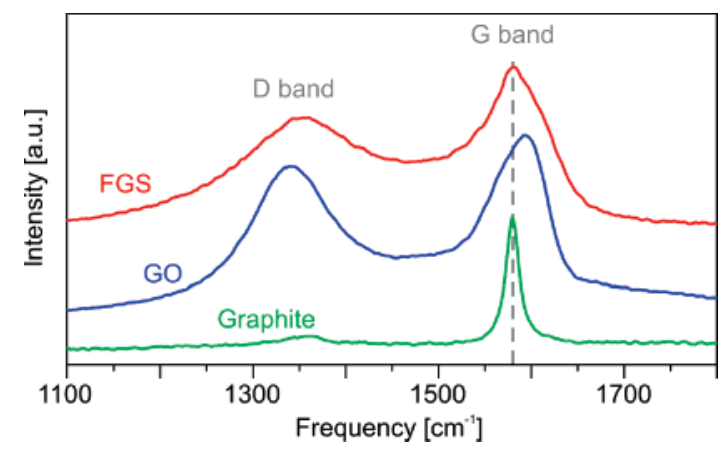

Figure 1. Evolution of the Raman spectra during the oxidation and exfoliation processes for graphite, GO, and FGS. All of the spectra correspond to an exciting laser wavelength of $514.5 \mathrm{~nm}$. The position of the $\mathrm{G}$ band peak in graphite is indicated by a vertical, dashed line.

Table 1: Raman Peak Positions and Left and Right Widths at Half-Maximum

\begin{tabular}{lccc}
\hline material & $\begin{array}{c}\text { LWHM } \\
{\left[\mathrm{cm}^{-1}\right]}\end{array}$ & $\begin{array}{c}\text { peak } \\
{\left[\mathrm{cm}^{-1}\right]}\end{array}$ & $\begin{array}{c}\text { RWHM } \\
{\left[\mathrm{cm}^{-1}\right]}\end{array}$ \\
\hline graphite & 9 & 1580 & 8 \\
GO & 55 & 1593 & 28 \\
FGS & 55 & 1581 & 43
\end{tabular}

of the graphite lattice (G band) at $1575 \mathrm{~cm}^{-1}$ as well as the (weak) disorder band caused by the graphite edges ( $\mathrm{D}$ band) at approximately $1355 \mathrm{~cm}^{-1} .^{12}$ (Raman spectra of samples were measured using a Renishaw 1000 microspectrometer using an excitation wavelength of $514.5 \mathrm{~nm}$. All samples were deposited on silicon wafers in powder form without using any solvent. The fluorescence background for the GO spectra was subtracted using the Wire 2.0 Renishaw software.) While the lattice vibration with the frequency of the $\mathrm{D}$ band is, in fact, present in the infinite graphene sheet, it remains Raman-inactive until the edges "light" it up due to the symmetry breaking and the corresponding change in the selection rules. Both the $G$ and the $D$ bands undergo significant changes upon amorphization of graphite as amorphous carbon contains a certain fraction of $\mathrm{sp}^{3}$ carbons. ${ }^{13}$ A universal observation is that higher disorder in graphite leads to a broader $\mathrm{G}$ band, as well as to a broad $\mathrm{D}$ band of higher relative intensity compared to that of the $\mathrm{G}$ band.

GO to FGS transformation leaves behind topological defects and vacancies. ${ }^{1,2}$ Among the vacancies, one of the more stable ones is the double vacancy $\left(\mathrm{C}_{2}\right)$, which is composed of two pentagonal rings and one octagonal ring and is usually referred to as the $5-8-5$ defect. ${ }^{14}$ In addition to such 5-8-5 defects, we have also investigated the common 5-7-7-5 rings, that is, the so-called Stone-Wales (SW) defects, which play an important role in carbon nanotubes and other graphitic materials. ${ }^{15}$ Along the graphiteGO-FGS path, the Raman spectra undergo changes (Figure 1) that are similar to those observed in the graphite to amorphous carbon transition. ${ }^{13}$ Specifically, the $G$ band broadens significantly and displays a shift to higher frequencies (blue-shift), and the D band grows in intensity. Table 1 indicates the peak positions of the $\mathrm{G}$ band in the materials we have investigated, as well as the corresponding left and right widths at half-maximum (LWHM and RWHM, respectively), which were calculated with respect to a baseline obtained by drawing a tangent line to the spectrum between 800 and $2,200 \mathrm{~cm}^{-1}$. A notable fact is that while the $\mathrm{G}$ band peak is located at a higher frequency in GO than that in graphite (1593 vs $1580 \mathrm{~cm}^{-1}$ ), in FGS, it is located almost at the same frequency as that in graphite. We stress that in all measurements, we have consistently used the same laser excitation frequency $(514.5 \mathrm{~nm})$. Thus, we can exclude spurious shifts due to changes in the laser frequency, which are known to affect the $\mathrm{D}$ band of graphite. ${ }^{16}$

As in the case of our Raman spectra of GO, the G band initially shifts to higher frequencies during graphite amorphization. ${ }^{13}$ A literature survey points to several possible explanations for this blue shift. First, in graphite with a sufficient concentration of defects, there is another band, the $\mathrm{D}^{\prime}$ band, located at $1620 \mathrm{~cm}^{-1},{ }^{12,17-19}$ which can partially merge with the $G$ band. The explanation given for the appearance of this band is that in graphite, there is a nonzero phonon density of states above the $\mathrm{G}$ band; such phonons, which are usually Raman-inactive, become active due to phonon confinement caused by the defects. ${ }^{19}$ Second, when going from a graphite crystal to a single graphene sheet, the $\mathrm{G}$ band shifts from 1581 to $1585 \mathrm{~cm}^{-1}$; this shift could also be partially responsible for the higher $\mathrm{G}$ band frequencies in GO if significant unmodified graphitic areas remain. ${ }^{20}$ Finally, isolated double bonds resonate at higher frequencies than the $\mathrm{G}$ band of graphite, ${ }^{13}$ although the specific patterns of the double bonds causing such an upshift, to the best of our knowledge, are not known. Compared to the G band of graphite, the blue-shifted peaks due to the first two causes are usually relatively weak. Therefore, the third alternative, namely, the presence of double bonds, appears to be the most plausible explanation of the blue shift of the $G$ band that we observed experimentally; we revisit this issue in great detail later in the paper.

In FGSs, the $\mathrm{G}$ band shifts back to the position (Figure 1) of the $\mathrm{G}$ band in graphite, which we attribute to a graphitic "self-healing" similar to what was observed from the sharpening of the G peak and the intensity decrease of the D peak in heat-treated graphite. ${ }^{21,22}$ In fact, in our STM experiments, we do observe the re-emergence of graphitic order in FGSs, as can be seen in Figure 2. (An amount of 5 $\mathrm{mg}$ of FGS was mixed with $5 \mathrm{~mL}$ of dimethylformamide (DMF) and ultrasonicated for $30 \mathrm{~min}$. The obtained dispersion was spin-cast onto a freshly cleaved sheet of highly oriented pyrolytic graphite (HOPG) at $5000 \mathrm{rpm}$. The sample was investigated by scanning tunneling microscopy (STM; Veeco MultiMode/NanoScope IIIa, Veeco Metrology LLC, Santa Barbara, CA, U.S.A.) using cut Pt/Ir tips at ambient conditions.) Compared to a STM image of highly oriented pyrolytic graphite (HOPG) taken under identical conditions (inset at the left bottom), FGS appears rough, featuring a peak-to-peak topography of $1 \mathrm{~nm}$. This roughness-due to functional groups and defects ${ }^{1}$ - prevents us from obtaining atomic resolution in all areas of the sheet. Nevertheless, the absolute value of the Fourier transform of the image (inset at the right top; hexagonal symmetry highlighted by manually 


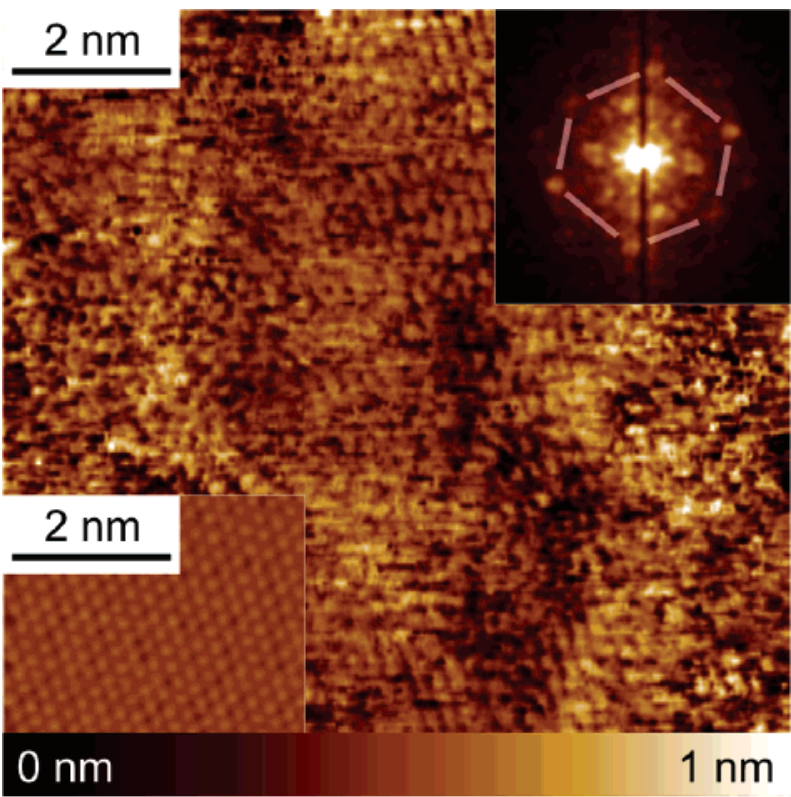

Figure 2. A $10 \times 10 \mathrm{~nm}$ STM topography image of a FGS, taken at a bias voltage of $30 \mathrm{mV}$ and a current of $5 \mathrm{nA}$. To minimize possible artifacts due to the size of the probe, the image was taken on top of a wrinkle of the sheet, where the topography is convex. The Fourier transform of the image shows that the hexagonal order is present (inset on the right top). The inset on the left bottom shows a STM image of HOPG taken under identical conditions (same topography color map as the main image).

added lines) shows a clear signature of a graphitic backbone. This indicates that the sheets undergo annealing during the reduction process. A recently proposed atomistic mechanism of graphite defect annealing can very well be responsible for such a behavior. ${ }^{23,24}$

The Raman spectra calculations were performed with the Quantum-Espresso (QE) package. ${ }^{25}$ Theoretical evaluation of the Raman spectra requires first-, second-, and third-order energy derivatives, among which the most demanding are the second-order derivatives of the energy with respect to the atomic positions (force constants) ${ }^{26}$ and the third-order polarizability derivatives, twice with respect to the electric field (yielding polarizability) and once with respect to the atomic positions (Raman intensity tensors). The important advantage of the $\mathrm{QE}$ code is that the polarizability derivatives have been recently implemented via a second-order response formalism, ${ }^{27}$ thus making their calculation relatively inexpensive compared to that of the force constants. To avoid the effect of the finite edges, we adopt a periodic supercell approach, using a $4 \times 4$ hexagonal supercell containing 16 graphene unit cells with 2 atoms each. In the third direction, we use a large enough spacing $(1.23 \mathrm{~nm})$ to make periodic image effects negligible. The resulting 32 carbon atom supercell is sufficient to investigate the 5-8-5 and 5-77-5 defects with reasonable isolation, as well as a variety of low-coverage oxidation products. In all of our calculations, we used the Becke-Lee-Yang-Parr (BLYP) exchangecorrelation functional ${ }^{28}$ and treated the valence-core interaction for the electrons with norm-conserving pseudopotentials. ${ }^{29}$ We simultaneously optimized the two lattice vectors in the plane and the atomic positions in the supercell by variable cell dynamics. ${ }^{30}$ The residual stress in the optimized structures did not exceed $300 \mathrm{MPa}$. In some cases, such as with highly nonplanar geometries or with $5-8-5$ and $5-7-$ 7-5 topological defects, there were significant changes $(\sim 5 \%)$ in the lattice vector lengths as a result of the optimization. To avoid asymmetric stresses, we placed the functional groups in pairs on the opposite sides of the sheet, with significant isolation from each other whenever possible. In this way, any buckling was symmetrically compensated. We used a plane wave expansion cutoff of $80 \mathrm{Ry}$ for the wave functions and of 320 Ry for the charge density in the fixed cell calculations, while higher cutoffs were employed in the variable cell calculations (95 and 380 Ry, respectively, using the modified kinetic energy functional introduced in ref 31). The Gamma point was used for the $k$-space integration. Notice that the Gamma point of the $4 \times 4$ graphitic supercell unfolds in the graphene unit cell into a $k$-mesh that excludes the $K$ point at the Brillouin zone boundary, where the valence and conduction bands of graphene are degenerate. This point would yield an infinite perturbative response in a calculation for an ideal graphene sheet. The Raman G peak for our 32 atom graphene supercell optimized with variable cell dynamics occurs at $1586 \mathrm{~cm}^{-1}$, in excellent agreement with the experimental value of 1585 $\mathrm{cm}^{-1}$ in single graphene sheets. ${ }^{20}$ In the following, we report atomic structure pictures, which were drawn with the help of the GaussView program. ${ }^{32}$ The Raman spectra corresponding to the various structures were broadened by convoluting the calculated Raman lines with a Gaussian having a fwhm of $12 \mathrm{~cm}^{-1}$.

First, following the GO model of Lerf et al., ${ }^{7}$ we calculated the Raman spectra of graphene with added oxygen-containing groups, both with the epoxy groups isolated (OX1 in Figure 3a) and arranged in a pair at the opposite edges of the same six-membered ring (OX2 in Figure $3 b)$. The latter structure was found to be a precursor for lattice unzipping ${ }^{33}$ in a previous study by our group. We also investigated the structures obtained by hydrolyzing the isolated epoxy groups, with two hydroxyls per pre-existing epoxy group. Here, we considered a pair of hydroxyl groups in the 1,2-position (ortho attachment in benzene) on the opposite sides of the sheet (OH1 in Figure 3c). In addition, we also investigated two nearby pairs of hydroxyl groups in the 1,4-positions (para attachment in benzene) such that an isolated double bond is formed $(\mathrm{OH} 2$ in Figure $3 \mathrm{~d}$, in which the atoms participating in the isolated double bond are shown in green).

The first noticeable effect of the addition of epoxy and hydroxyl groups (Figure 4) is a broadening of the graphite $\mathrm{G}$ band resulting from a series of new high-intensity bands that are red-shifted with respect to the $G$ band of pure graphite. In contrast, blue-shifted Raman-active bands can only be found in a structure with well-defined double bonds, albeit the intensity of such bands is low. Specifically, only the $\mathrm{OH} 2$ structure has such a peak due to the single isolated double bond. Second, the hydroxyl groups yield a series of bands around $1300 \mathrm{~cm}^{-1}$, which is exactly in the same range where the broad D band is located in the experimental data. Overall, due to the closeness in the frequencies of the $\mathrm{sp}^{3}$ 

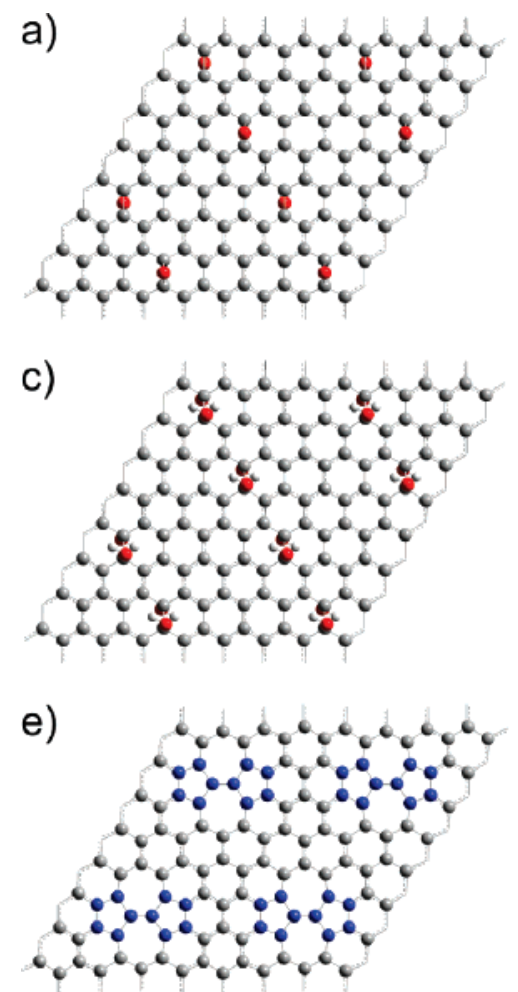
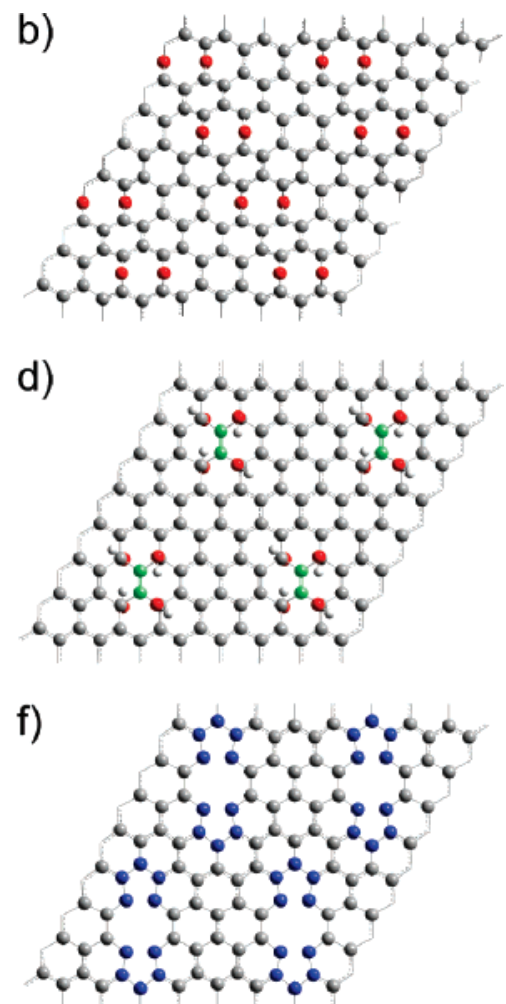

Figure 3. The $2 \times 2$ replicated cells with various groups and defects. Note that single, double, and conjugated bonds are drawn according to the distance criteria by GaussView..$^{32}$ (a) Graphene with individual epoxy groups (OX1). (b) Graphene with two epoxy groups arranged in a pre-unzipping pattern (OX2). (c) Graphene after hydrolysis, with a 1,2-hydroxyl pair per epoxy group (OH1). (d) Graphene with two 1,4-hydroxyl pairs $(\mathrm{OH} 2)$, forming an isolated double bond (contributing atoms colored green). (e) Graphene with the 5-7-7-5 defect (SW). (f) Graphene with a $\mathrm{C}_{2}$ vacancy (relaxed 5-8-5 defect structure).

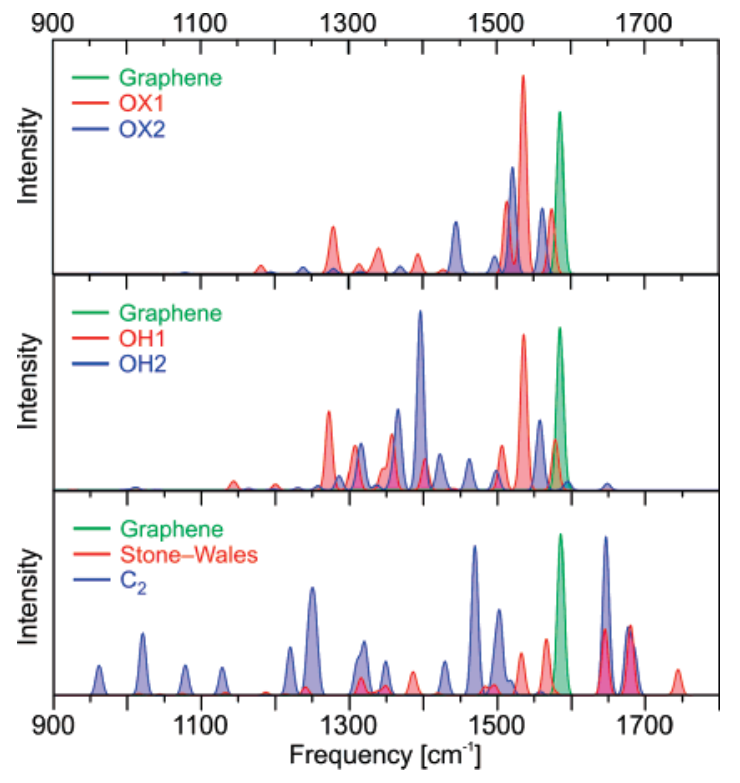

Figure 4. Calculated Raman spectra of graphene, $\mathrm{OX} 1, \mathrm{OX} 2, \mathrm{OH} 1$, $\mathrm{OH} 2, \mathrm{C}_{2}$ vacancy, and a SW defect.

carbon Raman-active band and the D band of graphite, both types of bands will tend to merge into a broad spectral feature.

The puzzling aspect of the Raman spectra of the model systems that we have considered so far is that none of the isolated and semi-isolated epoxy and hydroxyl groups produce any blue-shifted bands with high Raman intensity.
To obtain a high Raman intensity, a delocalized system of $\pi$-electrons is needed, and as we have seen in the example of the $\mathrm{OH} 2$ structure, isolated double bonds are insufficient in this respect. The Scholz-Boehm GO model ${ }^{5}$ (recently advocated by Szabo et al. ${ }^{34}$ ) contains $\mathrm{sp}^{2}$ ribbons with a rigid quinoidal structure (similar to R1 in Figure 5a). Since these ribbons are the narrowest possible, the double bonds are all isolated. However, if ribbons of this type have more than just one layer of $\mathrm{sp}^{2}$ carbons, they develop a well-defined pattern of alternating single and double bonds within the interconnected $\mathrm{sp}^{2}$ carbon region, displayed as $\mathrm{R} 2$ in Figure 5b. In R2, the calculated Raman-active band is at $1620 \mathrm{~cm}^{-1}$, that is, blue-shifted compared to pure graphite (Figure 5d). Moreover, because of the interconnected $\mathrm{sp}^{2}$ carbons, $\pi$-electrons are delocalized within the ribbon, and the Raman intensity of the blue-shifted band is high, in contrast to R1. Removal of half of the hydroxyl groups from such a structure results in the structure indicated as R3 in Figure 5c. In R3, we observe that the single-double bond length alternation disappears with a subsequent shift of the $\mathrm{G}$ band back to graphitic values. The Scholz-Boehm structure itself, R1, does have a Raman-active band due to isolated double bonds at a relatively high frequency of $1690 \mathrm{~cm}^{-1}$, albeit with a low intensity due to the disappearance of the chains of $\mathrm{sp}^{2}$ carbon atoms.

Although the R2 structure has the blue-shifted peak observed in the experiment, it also has another peak of high intensity at $1390 \mathrm{~cm}^{-1}$ which is not found in the experimental 

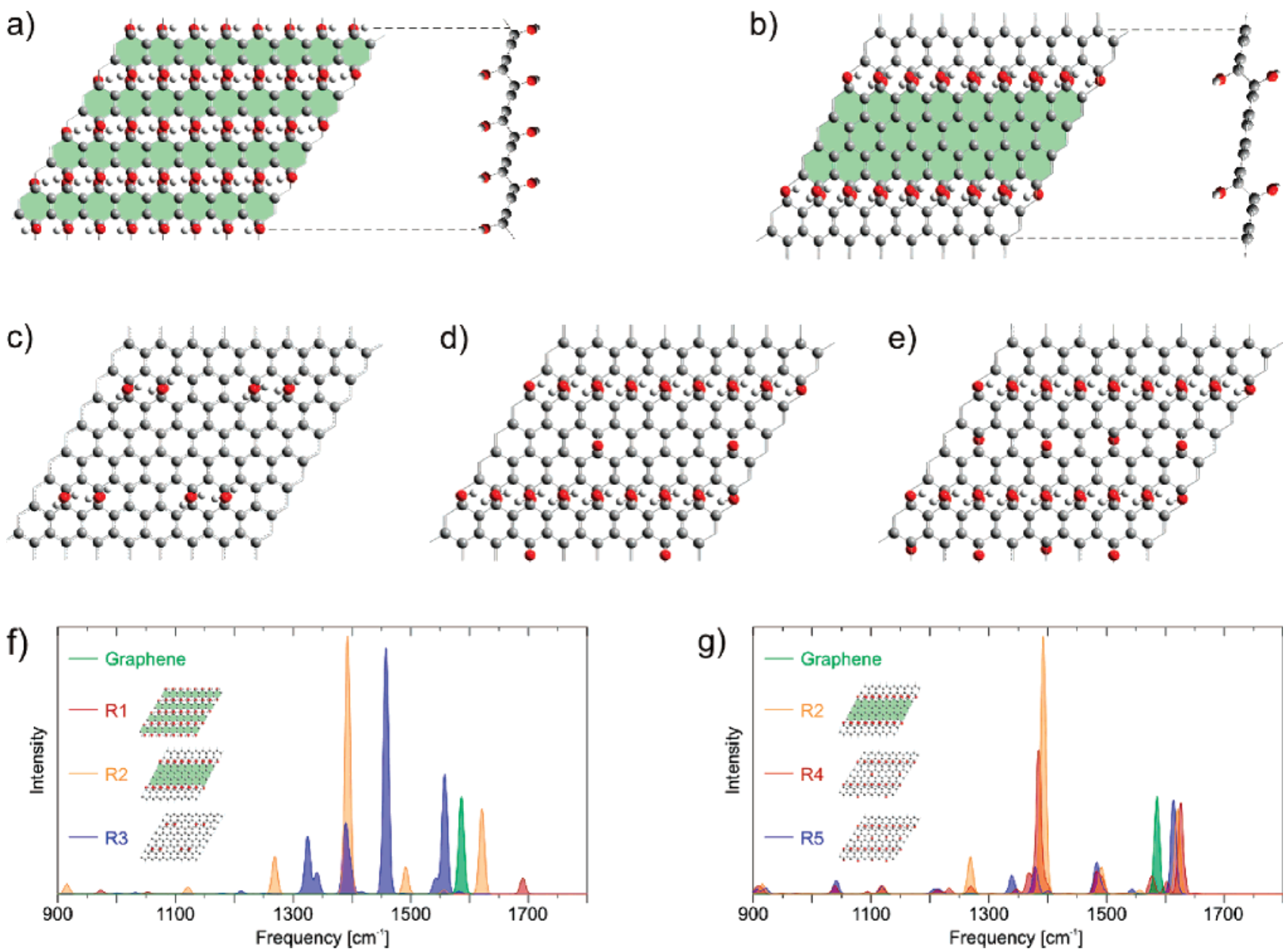

Figure 5. (a) GO with the narrowest ribbons (R1), as per the Scholz-Boehm model (see text), (b) GO with wider ribbons (R2), and (c) GO without ribbons (R3). The hexagons that are part of the ribbons are highlighted in green. (d) R2 structure with an additional epoxy group in the middle of the ribbon (R4). (e) R2 structure with two additional epoxy groups (R5). (f and g) The calculated Raman spectra are shown, with intensities for R1 and R2 multiplied by 10 and 0.5 , respectively. Note that in panels a-e the $2 \times 2$ replicated computational cells are shown, and single, double, and conjugated bonds are drawn according to the distance criteria by GaussView. ${ }^{32}$

data. Furthermore, the R2 structure does not have enough oxygen atoms to match the $\mathrm{C} / \mathrm{O}$ ratio $(2 / 1)$ measured for $\mathrm{GO}$. To address both of these concerns, we have investigated the structures R4 and R5 that have additional epoxy groups in the middle of the $\mathrm{sp}^{2}$ ribbon with respect to $\mathrm{R} 2$. Both of these structures can be viewed as intermediates on the path from R2 to R1. By hydrolysis and addition of extra epoxy/ hydroxyl groups, R4 and R5 would become R1. Remarkably, in these new structures, we find that the intensity of the peak at $1390 \mathrm{~cm}^{-1}$ decreases substantially, while the blue-shifted peak is preserved at roughly the same frequency, thus yielding a much better overall agreement with the experimental spectra in Figure 1.

An important comment is related to the resulting thickness of the carbon sheet due to these types of functionalization. As the side views of the structures in Figure 5 indicate, the ribbons introduce out-of-plane zigzag distortions into the carbon lattice. The heights of these steps are 90, 120, and $100 \mathrm{pm}$ for the structures R1, R2, and R3, respectively. Therefore, in all cases, the effect of the carbon lattice "steps" on the GO profile is negligible, and it is mostly the sidegroups that yield the $0.7 \mathrm{~nm}$ thickness in GO.
Finally, we have investigated the Raman spectra of a SW (5-7-7-5) defect (Figure $3 \mathrm{e})^{15}$ and a $\mathrm{C}_{2}$ vacancy $(5-8-5)$ defect (Figure 3f). ${ }^{14}$ As noted earlier, in these cases, the supercell does change quite significantly upon relaxation. The $\mathrm{C}_{2}$ vacancy causes a significant reduction in the lattice vectors, which is not surprising since 2 out of 32 carbon atoms are removed. Although the optimized 5-8-5 defect does not have any local curvature and within the $2 \mathrm{D}$ supercell stays completely flat, we found, in preliminary tests, that the defect is more stable on curved surfaces such as, for example, nanotubes. Therefore, if present in sufficient concentrations, these defects might be able to induce curvature in small flakes like those studied in ref 1 . Specifically, in small flakes, the curvature would be determined by the balance of the energy gain due to the $5-8-5$ defect relaxation and the energy loss due to the strain induced on the nearby graphitic carbons. Overall, the Raman spectra for the $\mathrm{C}_{2}$ vacancy and the SW defect have a lot of new features, with the notable fact that there is a series of bands above the $\mathrm{G}$ band of graphite. Specifically, the $\mathrm{C}_{2}$ vacancy has Raman peaks at 1647,1676 , and $1686 \mathrm{~cm}^{-1}$ (the latter two almost merge in the figure), whereas the SW defect has 
peaks at 1645 and $1680 \mathrm{~cm}^{-1}$, as well as at $1744 \mathrm{~cm}^{-1}$. It is worth noting that our calculations yield Raman peaks in the vicinity of 1645 and $1680 \mathrm{~cm}^{-1}$ for both structures. Upon examination of the atoms participating in these normal modes, we find that these vibrations derive from the displacements of the carbon atoms which belong simultaneously to five- and six-membered rings, a configuration shared by both structures. Thus, the defects are also capable of producing blue shifts. However, the experimental data do not show significant Raman intensities above $1650 \mathrm{~cm}^{-1}$ for both GO and FGS, suggesting that the concentration of such defects in both materials should not be very high. In the case of GO, this is not surprising since a plausible mechanism for the appearance of such defects in large numbers during the oxidation process is not known at the moment. In FGSs, in contrast, the emergence of such defects through carbon emission during reduction is probable. ${ }^{1}$ The absence of a Raman signal in the corresponding spectral range, however, suggests that many of these defects may have been removed through annealing. The remaining ones may be concentrated in the few areas where the sheets are wrinkled, as their presence has been associated with curved structures. ${ }^{1}$

In summary, in our experimental work on the transformation from graphite to GO, one of the most puzzling aspects is the blue shift of the graphitic $\mathrm{G}$ band in graphite oxide while a reversal is observed after partial reduction to produce FGSs. Computationally, among all of the structures that we have considered, only the alternating pattern of singledouble carbon bonds within the $\mathrm{sp}^{2}$ carbon ribbons as well as the SW and the 5-8-5 defect structures yields Raman bands of high intensity that are blue-shifted compared to the $\mathrm{G}$ band of graphite. While isolated double bonds can also lead to blue-shifted bands, the Raman intensity of such bands is significantly lower. To obtain single-double bond alternation within extended $\mathrm{sp}^{2}$ carbon areas, it is necessary to have $\mathrm{sp}^{3}$ carbons on the edges of a zigzag carbon ribbon. The Scholz-Boehm GO model introduced the narrowest ribbons of this type; however, in such narrow ribbons the double bonds are all isolated, yielding low blue-shifted Raman intensities in correspondence with the double bonds. The structures that yield the best match with experiment have wider ribbons, as we advocate, with some additional epoxy groups in the middle. The out-of-plane distortions introduced to the carbonaceous backbone by the structures investigated here are around $0.1 \mathrm{~nm}$, implying that the sheets essentially remain flat. One of the most significant findings of this work is that a novel GO model containing areas of $\mathrm{sp}^{2}$ carbons with an alternating pattern of single-double carbon bonds is able to explain the experimentally observed blue $\mathrm{G}$ band shift in GO.

Acknowledgment. Financial support from the National Science Foundation NIRT under Grant No. CMS-0609049 and the NASA University Research, Engineering, and Technology Institute on BioInspired Materials (BIMat) under Award No. NCC-1-02037 is greatly appreciated. We acknowledge the use of the Materials Characterization Facility at Drexel University for Raman spectroscopy. A computer time grant from the TIGRESS High Performance Computing Center at Princeton University is also acknowledged.

\section{References}

(1) Schniepp, H. C.; Li, J.-L.; McAllister, M. J.; Sai, H.; Herrera-Alonso, M.; Adamson, D. H.; Prud'homme, R. K.; Car, R.; Saville, D. A.; Aksay, I. A. J. Phys. Chem. B 2006, 110, 8535.

(2) McAllister, M. J.; Li, J.-L.; Adamson, D. H.; Schniepp, H. C.; Abdala, A. A.; Liu, J.; Herrera-Alonso, M.; Milius, D. L.; Car, R.; Prud'homme, R. K.; Aksay, I. A. Chem. Mater. 2007, 19, 4396.

(3) Hofmann, U.; Holst, R. Ber. Dtsch. Chem. Ges. 1939, 72, 754.

(4) Ruess, G. Monatsh. Chem. 1946, 76, 381.

(5) Scholz, W.; Boehm, H. P. Z. Anorg. Allg. Chem. 1969, 369, 327.

(6) Nakajima, T.; Mabuchi, A.; Hagiwara, R. Carbon 1988, $26,357$.

(7) Lerf, A.; He, H. Y.; Forster, M.; Klinowski, J. J. Phys. Chem. B 1998, 102, 4477.

(8) Kohn, W.; Sham, L. J. Phys. Rev. 1965, 140, A1133.

(9) Rinzler, A. G.; Liu, J.; Dai, H.; Nikolaev, P.; Huffman, C. B.; Rodriguez-Macias, F. J.; Boul, P. J.; Lu, A. H.; Heymann, D.; Colbert, D. T.; Lee, R. S.; Fischer, J. E.; Rao, A. M.; Eklund, P. C.; Smalley, R. E. Appl. Phys. A 1998, 67, 29.

(10) Irle, S.; Mews, A.; Morokuma, K. J. Phys. Chem. A 2002, 106, 11973.

(11) Fuente, E.; Menendez, J. A.; Diez, M. A.; Suarez, D.; Montes-Moran, M. A. J. Phys. Chem. B 2003, 107, 6350.

(12) Tuinstra, R.; Koenig, J. L. J. Chem. Phys. 1970, 53, 1126.

(13) Ferrari, A. C.; Robertson, J. Phys. Rev. B 2000, 61, 14095

(14) Lee, G. D.; Wang, C. Z.; Yoon, E.; Hwang, N. M; Kim, D. Y.; Ho, K. M. Phys. Rev. Lett. 2005, 95, 205501.

(15) Stone, A. J.; Wales, D. J. Chem. Phys. Lett. 1986, 128, 501.

(16) Kawashima, Y.; Katagiri, G. Phys. Rev. B 1995, 52, 10053.

(17) Nemanich, R. J.; Solin, S. A. Phys. Rev. B 1979, 20, 392.

(18) Livneh, T.; Haslett, T. L.; Moskovits, M. Phys. Rev. B 2002, 66, 195110.

(19) Cancado, L. G.; Pimenta, M. A.; Neves, B. R. A.; Dantas, M. S. S.; Jorio, A. Phys. Rev. Lett. 2004, 93, 247401.

(20) Graf, D.; Molitor, F.; Ensslin, K.; Stampfer, C.; Jungen, A.; Hierold, C.; Wirtz, L. Nano Lett. 2007, 7, 238.

(21) Han, C. C.; Lee, J. T.; Chang, H. Chem. Mater. 2001, 13, 4180.

(22) Sato, K.; Saito, R.; Oyama, Y.; Jiang, J.; Cancado, L. G.; Pimenta, M. A.; Jorio, A.; Samsonidze, G. G.; Dresselhaus, G.; Dresselhaus, M. S. Chem. Phys. Lett. 2006, 427, 117.

(23) Ding, F.; Jiao, K.; Wu, M. Q.; Yakobson, B. I. Phys. Rev. Lett. 2007, 98, 075503.

(24) Ding, F.; Jiao, K.; Lin, Y.; Yakobson, B. I. Nano Lett. 2007, 7, 681.

(25) Quantum Espresso. http://www.quantum-espresso.org.

(26) Baroni, S.; de Gironcoli, S.; Dal Corso, A.; Giannozzi, P. Rev. Mod. Phys. 2001, 73, 515.

(27) Lazzeri, M.; Mauri, F. Phys. Rev. Lett. 2003, 90, 036401.

(28) (a) Becke, A. D. Phys. Rev. A 1988, 38, 3098. (b) Lee, C. T.; Yang, W. T.; Parr, R. G. Phys. Rev. B 1988, $37,785$.

(29) Hamann, D. R.; Schluter, M.; Chiang, C. Phys. Rev. Lett. 1979, 43, 1494.

(30) Wentzcovitch, R. M.; Martins, J. L.; Price, G. D.; Phys. Rev. Lett. 1993, 70, 3947.

(31) Bernasconi, M.; Chiarotti, G. L.; Focher, P.; Scandolo, S.; Tosatti, E.; Parrinello, M. J. Phys. Chem. Solids 1995, 56, 501.

(32) Dennington, R., II; Keith, T.; Millam, J.; Eppinnett, K.; Hovell, W. L.; Gilliland, R. GaussView, version 3.09; Semichem, Inc.: Shawnee Mission, KS, 2003.

(33) Li, J.-L.; Kudin, K. N.; McAllister, M. J.; Prud'homme, R. K.; Aksay, I. A.; Car, R. Phys. Rev. Lett. 2006, 96, 176101.

(34) Szabo, T.; Berkesi, O.; Forgo, P.; Josepovits, K.; Sanakis, Y.; Petridis, D.; Dekany, I. Chem. Mater. 2006, 18, 2740. 